\title{
Plasmonic Nanocavities Enable Self-Induced Electrostatic Catalysis**
}

\author{
Clàudia Climent,* Javier Galego, Francisco J. Garcia-Vidal, and Johannes Feist*
}

\begin{abstract}
The potential of strong interactions between light and matter remains to be further explored within a chemical context. Towards this end herein we study the electromagnetic interaction between molecules and plasmonic nanocavities. By means of electronic structure calculations, we show that selfinduced catalysis emerges without any external stimuli through the interaction of the molecular permanent and fluctuating dipole moments with the plasmonic cavity modes. We also exploit this scheme to modify the transition temperature $T_{1 / 2}$ of spin-crossover complexes as an example of how strong lightmatter interactions can ultimately be used to control a materials responses.
\end{abstract}

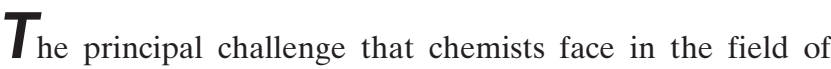
nanoscience is to be able to manipulate individual molecules. This is a key point in order to ultimately control the properties and response of materials as well as the course and outcome of chemical reactions. In the last decade, novel ways of altering chemical reactions have been developed. For example, mechanochemistry aims to shape the potential energy surface (PES) connecting reactants to products by applying a mechanical force. ${ }^{[1-4]}$ Another example, closer to the strategy we propose herein, is to apply external electric fields to catalyze or inhibit chemical reactions. This was computationally predicted by Shaik and co-workers ${ }^{[5,6]}$ and recently achieved experimentally for a Diels-Alder reaction, ${ }^{[7]}$ boosting the field of electrostatic catalysis. ${ }^{[8,9]}$ It is important to highlight that prior to these works, much effort was spent to elucidate the contribution of electrostatics to enzymatic catalysis, ${ }^{[10]}$ lately inspiring the optimization of catalysts by mimicking the electrostatic environmental effect of the protein scaffold. ${ }^{[11]}$ Plasmonic catalysis is another field popularized in recent years because of the ability of hot carriers to lower reaction barriers and enable new reaction pathways. ${ }^{[12-16]}$

[*] Dr. C. Climent, J. Galego, Prof. Dr. F. J. Garcia-Vidal, Dr. J. Feist Departamento de Física Teórica de la Materia Condensada and Condensed Matter Physics Center (IFIMAC)

Universidad Autónoma de Madrid

28049 Madrid (Spain)

E-mail: claudia.climent@uam.es johannes.feist@uam.es

[***] A previous version of this manuscript has been deposited on a preprint server (https://doi.org/10.26434/chemrxiv.7716254.v1).

(2) Supporting information and the ORCID identification number(s) for

(iD the author(s) of this article can be found under: https://doi.org/10.1002/anie.201901926.

of (c) 2019 The Authors. Published by Wiley-VCH Verlag GmbH \& Co. $\mathrm{KGaA}$. This is an open access article under the terms of the Creative Commons Attribution-NonCommercial License, which permits use, distribution and reproduction in any medium, provided the original work is properly cited and is not used for commercial purposes.
Herein we propose an alternative strategy to catalyze chemical reactions, tackling the situation of strong coupling between plasmonic modes (collective oscillations of free electrons in a metal) and molecular dipole moments, without the need of exciting the system with a laser nor applying an external electric field. This can be achieved, for instance, with the bowtie configuration shown in Figure 1, in which the highly confined electromagnetic mode within the antenna gap can strongly interact with the molecules in that region. ${ }^{[17]}$ In the following we will exemplify this effect with classical examples of $\mathrm{S}_{\mathrm{N}} 1$ and $\mathrm{S}_{\mathrm{N}} 2$ reactions, which have also been studied in electrostatic catalysis. ${ }^{[18-20]}$ In addition, our proposal can also be exploited to modify molecular properties, as we illustrate with spin-crossover transitions in a series of $\mathrm{Fe}^{\mathrm{II}}$ complexes.

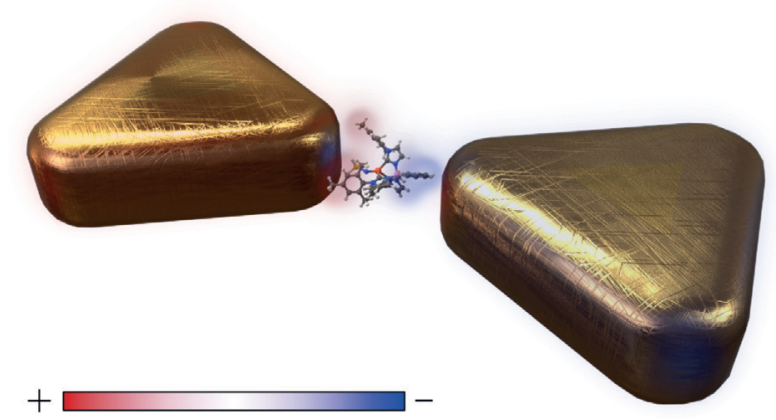

Figure 1. Sketch of a configuration to enable self-induced electrostatic catalysis consisting of a molecule within the gap of a metallic bowtie nanocavity. The charge distribution of the molecule and the one induced in the metal is also illustrated through red and blue shading.

When molecular excitations and electromagnetic (EM) modes strongly interact, hybrid light-matter states known as polaritons are formed. The confined light modes necessary to produce these new eigenmodes of the system may be hosted in Fabry-Perot or plasmonic nanocavities. ${ }^{[21]}$ Polaritonic chemistry ${ }^{[22-25]}$ is an emerging field aiming to manipulate the behavior of molecules with the formation of polaritons. In particular, Ebbesen and co-workers have reported two prime examples of polaritonic chemistry in Fabry-Perot cavities, experimentally demonstrating the suppression of ground ${ }^{[26,27]}$ and excited state ${ }^{[28]}$ reactions taking advantage of polaritons. Examples of photo-oxidation and photodegradation under strong coupling have also been reported very recently. ${ }^{[29,30]}$

We recently tackled from a theoretical point of view the coupling of one or several EM modes in a nanocavity to thermally driven chemical reactions in the ground state. ${ }^{[31]}$ In the present work we have implemented and interfaced this theory with quantum chemistry calculations. In the following we summarize the main points of the theory for completeness. 
Starting from a purely quantum mechanical description of the coupled light-matter system, we found a novel phenomenon, different to the examples described above, which can be exploited to alter ground state chemical reactivity and molecular properties that 1 ) does not require the formation of polaritons (i.e. independent of resonance), 2) has a clear interpretation in terms of Van der Waals or Casimir-Polder interactions, and 3) is a single-molecule and not a collective effect. As a result of the interaction between the molecular dipole of the species involved in the reaction and the induced dipole in the nanocavity, the ground-state PES is modified according to the square of the molecular dipole moment. Note that, as opposed to the external electric field in the Stark effect, the field in the present case is generated by the molecule itself by light (i.e. quantized EM modes)-matter interactions.

We used the following Hamiltonian in the cavity BornOppenheimer approximation ${ }^{[32]}$ (CBOA) to investigate the coupling of a molecular dipole to $k$ cavity modes [Eq. (1)]

$$
\hat{H}_{e}(\widehat{\boldsymbol{x}} ; \boldsymbol{R}, q)=\hat{H}_{e}(\widehat{\boldsymbol{x}} ; \boldsymbol{R})+\frac{1}{2} \sum_{k} \omega_{k}^{2} q_{k}^{2}+\sum_{k} \omega_{k} q_{k} \lambda_{k} \widehat{\mu}(\widehat{\boldsymbol{x}} ; \boldsymbol{R})
$$

The first term is the standard electronic Born-Oppenheimer Hamiltonian, the second one is the potential energy of the quantized electromagnetic (EM) modes, which are modeled as harmonic oscillators, and the third term represents the light-matter interaction. The frequency of the EM modes is given by $\omega_{k}, q_{k}$ are the photonic displacements, related to the electric field of each mode, and the $\lambda_{k}$ coupling strengths quantify the light-matter interaction along the direction of the molecular dipole. The molecular dipole moment operator is given by $\widehat{\mu}$ while $\mathbf{R}$ and $\widehat{\boldsymbol{x}}$ refer to the nuclear and electronic positions, respectively. Within the CBOA, the cavity modes are additional nuclear-like degrees of freedom parametrized by $q_{k}$, and thus one can benefit from the standard approaches available in quantum chemistry to optimize minima and transition states (Supporting information). Note that this has been implemented in the present work. For the minimum energy path along all the $q_{k}$ in the CBOA and within a single-molecule level, an effective coupling constant $\lambda$ can be defined as $\sqrt{\sum_{k} \lambda_{k}^{2}}$ together with an effective frequency $\omega$ given by $\sum_{k} \lambda_{k}^{2} \omega_{k} / \lambda^{2}$ and an effective mode volume $V=1 / \varepsilon_{0} \lambda$ to which several modes contribute. Note that the magnitude of this coupling strength will depend on the considered plasmonic structure.

Up to second order of perturbation theory, the energy shift on the PES when the molecular dipole couples to $k$ modes of a plasmonic nanocavity can be simply written as Equation $(2)^{[31]}$

$$
\delta E(\mathbf{R})=-\frac{\lambda^{2}}{2}\left(\mu_{o}^{2}(\mathbf{R})+\frac{\omega}{2} \alpha_{0}(\mathbf{R})\right)
$$

This is an important result showing that the profiles of the ground state permanent dipole moment $\mu_{0}(\mathbf{R})$ and the static polarizability $\alpha_{0}(\mathbf{R})$ dictate how the energy landscape is modified. It is worth noticing that this shift corresponds to the well-known Casimir-Polder interaction energy between neu- tral atoms and macroscopic conducting bodies arising from the vacuum fluctuations of the EM field. ${ }^{[33,34]}$ This was derived by Casimir and Polder within a quantum electrodynamics framework (QED), motivated by the discrepancies between theory and experimental results on colloidal particle stability, and led to a generalization of Van der Waals interactions. ${ }^{[35-37]}$ This connection leads to a straightforward interpretation of Equation (2). In particular, if the cavity can be approximated by a point dipole, which would be valid for instance for a small enough plasmonic nanoparticle, then the two energy shifts in the PES of Equation (2) represent: 1) the Debye energy of the Van der Waals interaction between the permanent dipole of the molecule and the dipole it induces in the nanoparticle and 2) the interaction between fluctuating dipoles in the molecule and nanoparticle expressed by the London force. Note that the Debye term will always lower the energy in contrast to the London one which can act in both directions. For all the examples we have studied, the Debye term always dominates the energy shift.

We first focus on how coupling to a plasmonic nanocavity can lead to changes in ground-state chemical reactivity. We have chosen the $S_{N} 1$ and $S_{N} 2$ reactions since the permanent dipole moment along their reaction paths varies considerably, thus making them ideal candidates to demonstrate chemical changes in the present QED scenario. We consider the hydrolysis of tert-butyl chloride, which is known to proceed via an $\mathrm{S}_{\mathrm{N}} 1$ mechanism. ${ }^{[38]}$ This reaction consists of three steps: 1) the separation of the leaving group leading to a carbocationic intermediate, 2) solvent nucleophilic attack on the carbocation, and 3) deprotonation leading to an alcohol and $\mathrm{HCl}$. Steps (2) and (3) are known to be fast, and thus we focus herein on the rate-determining step (1). In Figure 2 we show the reaction profile for different coupling strengths. In realistic multi-mode nanocavities, for example, nanoparticle on a mirror setup, the effective global coupling constant can reach values of more than 0.02 atomic units (a.u. $)^{[31]}(V=$ $5 \mathrm{~nm}^{3}$ ), thus we have herein considered $\lambda$ coupling strengths

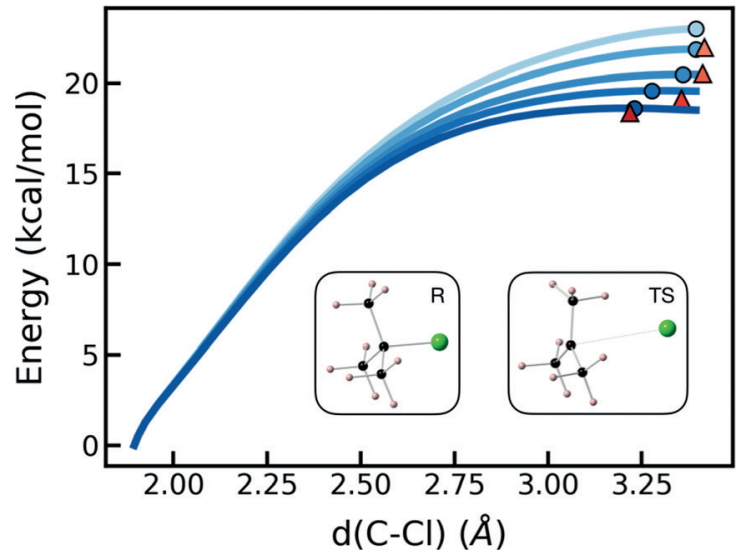

Figure 2. Reaction profile for the initial step of the $S_{N} 1$ reaction involving leaving group separation of tert-butyl chloride. Increasing color darkness corresponds to increasing coupling strength $(\lambda=0$, $0.01,0.015,0.0175,0.02$ a.u.). Blue circles correspond to the TS within perturbation theory while red triangles indicate the optimized TS obtained with a self-consistent electric field. Reactant (R) and transition state (TS) structures shown for the bare case, $\mathrm{Cl}$ green, $\mathrm{C}$ black. 
of up to this value. Note that at very short distances, a metal surface could also provide strong light-matter coupling but with a smaller coupling strength.

Due to the separation of charge during the heterolytic fission, the dipole moment increases along the path from the reactant to the transition state (TS), with largest modifications of the reaction profile occurring near the TS. The barrier is significantly reduced by approximately $4 \mathrm{kcal} \mathrm{mol}^{-1}$ (with $\lambda=0.02$ a.u.) representing about $20 \%$ of the bare energy barrier due to stabilization of the TS by the cavity mode, exclusively due to the Debye interaction. Another important result is that the position of the TS (circles in plot) is shifted to shorter $\mathrm{C}-\mathrm{Cl}$ bond lengths as the light-matter interaction increases, a fingerprint of the change in electronic structure. Since these results are based on Equation (2), which was derived from perturbation theory, we checked our results by performing direct optimization on the CBOA surface defined by Equation (1) (triangles in Figure 2). Both results are in very good agreement, proving the suitability of Equation (2) to calculate energy shifts when a molecule is placed in a plasmonic nanocavity and confirming that TS structures can be modified in such a scenario. In particular, the $\mathrm{C}-\mathrm{Cl}$ bond length of the TS is shortened by about $0.18 \AA$ and one of the $\mathrm{C}-\mathrm{C}-\mathrm{Cl}$ angles increases by $13^{\circ}$ for $\lambda=0.02$ a.u. as compared to the case with no coupling (Supporting information).

In the Menshutkin reaction, ${ }^{[39]}$ an ammonia molecule attacks the carbon center in methyl chloride, leading to a pentacoordinate TS, with an energy barrier of around $13 \mathrm{kcal} \mathrm{mol}^{-1}$ and eventual formation of an ion-pair product complex. In this case the dipole moment also increases along the course of the reaction, thus the greatest modifications on
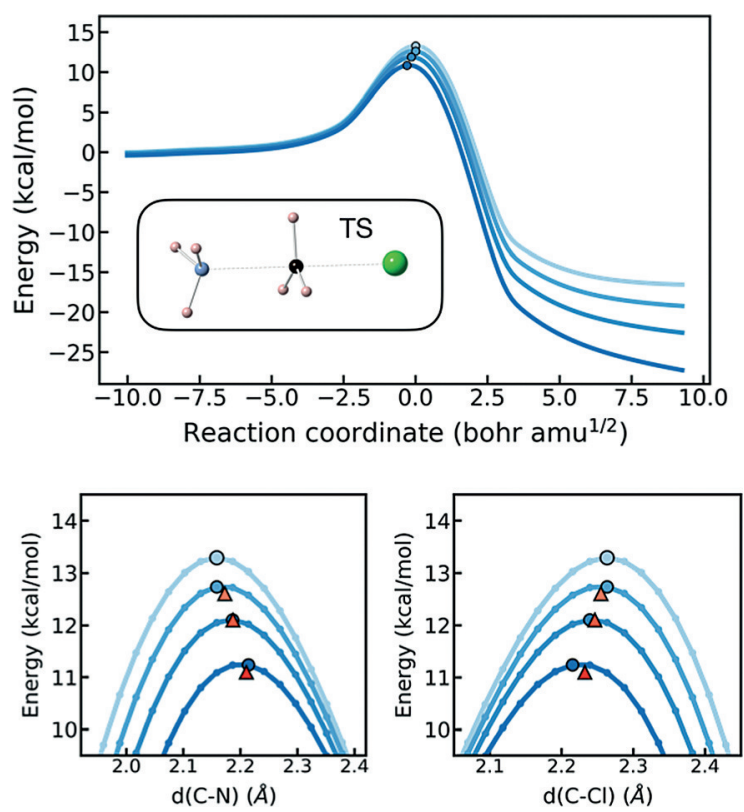

Figure 3. Profile (top) for the $\mathrm{S}_{\mathrm{N}} 2$ reaction. Increasing color darkness corresponds to increasing coupling strength $(\lambda=0,0.01,0.015$, 0.02 a.u.). Expansion of the barrier region (bottom) plotted along the $d(\mathrm{C}-\mathrm{N})$ and $d(\mathrm{C}-\mathrm{Cl})$ bond lengths. Blue circles correspond to the TS within perturbation theory while red triangles indicate the optimized TS obtained with a self-consistent electric field. The TS geometry is shown for the bare case. the reaction profile are observed from the TS onwards, as shown in Figure 3. The energy barrier decreases by about $20 \%$ for $\lambda=0.02$ a.u., with the Debye interaction contributing $84 \%$ and the London one accounting for $16 \%$. Although the London force does not dominate the change in barrier, it serves to illustrate that in cases where the molecular polarizability varies along the reaction path, London interactions can play an important role. By means of the proposed selfinduced effect, note also that the product complex in this reaction is greatly stabilized relative to the reactants by the presence of the nanocavity, making the reaction more exothermic. This suggests that the competition between forward and backward processes in some reactions may also be altered in such a scenario.

Serving as an example of manipulation of molecular properties in the present QED scenario, in the following we show how control over the spin states of spin-crossover ${ }^{[40]}$ (SCO) complexes and their characteristic transition temperature $T_{1 / 2}$ can be also achieved. In these systems, the electronic energies of two spin states are close enough that a transition between them can be thermally induced. This is because the high-spin (HS) state is entropically favored, and is thus the one preferred at higher temperatures while the low-spin (LS) state prevails at lower ones, and at $T_{1 / 2}$, both spin states are equally populated. We propose coupling to a nanocavity mode as a novel way to control the relative energy of the LS and HS states in transition metal complexes and thus modulate their SCO behavior. To illustrate this, we have considered four-coordinated $\mathrm{Fe}^{\mathrm{II}}$ phosphoraminimato complexes of general formula $\mathrm{PhB}(\mathrm{MesIm})_{3} \mathrm{Fe}=\mathrm{PR}_{3}$ with $\mathrm{R}=$ $\mathrm{H}, \mathrm{Me},{ }^{n} \mathrm{Pr}, \mathrm{Ph}_{3}$ (Figure 4) that undergo a thermally induced

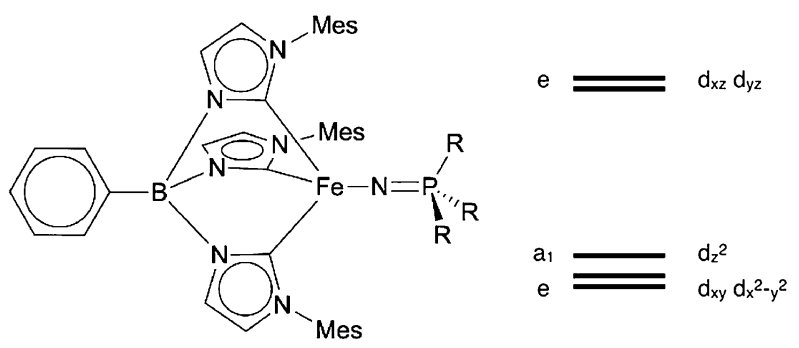

Figure 4. Chemical structure of the $\mathrm{Fe}^{\prime \prime}$ complexes with $\mathrm{R}=\mathrm{H}, \mathrm{Me},{ }^{n} \mathrm{Pr}$, $\mathrm{Ph}$. Schematic diagram of the d-orbital splitting corresponding to $C_{3 v}$ point group symmetry.

$S=0$ to $S=2$ spin-crossover transition in solution, and for which a correlation between $T_{1 / 2}$ and the size of the phosphine ligand was found. ${ }^{[41]}$ The intimate relation between the electronic structure and the corresponding SCO behavior was analyzed in further detail in a recent computational study. ${ }^{[42]}$ Note that switching between the spin states of similar systems has been achieved in single-molecule junctions. ${ }^{[43]}$

As shown in Table 1, the computed electronic energies of the LS and HS states of the Fe ${ }^{\mathrm{II}}$ complexes are close enough, within 2-6 kcal mol${ }^{-1}$, a necessary condition to enable SCO. By considering the $\Delta G$ temperature dependence, the $T_{1 / 2}$ values were estimated (Supporting Information), reproducing the already observed trend of decreasing $T_{1 / 2}$ with increasing phosphine size. ${ }^{[41,42]}$ 
Table 1: Energy difference between the low and high spin state of the studied Fe" complexes together with their dipole moment and the experimental and estimated SCO transition temperature.

\begin{tabular}{llllllll}
\hline $\mathrm{R}$ & $\begin{array}{l}\mathrm{E}_{\mathrm{LS}}-\mathrm{E}_{\mathrm{HS}} \\
{\left[\mathrm{kcalmol}^{-1}\right]}\end{array}$ & \multicolumn{2}{c}{$\mu[\mathrm{D}]$} & & \multicolumn{3}{c}{$T_{1 / 2}[\mathrm{~K}]$} \\
& & $\mathrm{HS}$ & $\mathrm{LS}$ & Exp. & 0.00 & $0.02^{[\mathrm{a}]}$ & $0.02^{[\mathrm{b}]}$ \\
\hline $\mathrm{H}$ & -5 & 2.05 & 4.60 & - & 392 & 417 & 423 \\
$\mathrm{Me}$ & -6 & 3.21 & 6.20 & 340 & 351 & 384 & 396 \\
${ }^{\mathrm{n}} \mathrm{Pr}$ & -4 & 3.05 & 6.26 & 214 & 192 & 226 & 237 \\
$\mathrm{Ph}$ & -2 & 2.31 & 5.56 & 81 & 74 & 98 & 103
\end{tabular}

[a] $\mu_{\mathrm{HS}}$ and $\mu_{\mathrm{LS}}$ aligned with the polarization of the plasmonic mode. [b] $\mu_{\mathrm{LS}}$ aligned with the polarization of the plasmonic mode and perpendicular to $\mu_{\mathrm{HS}}$

a Ramón y Cajal grant (JF). We also acknowledge support by the QuantERA program of the European Commission with funding by the Spanish AEI through project PCI2018-093145.

\section{Conflict of interest}

The authors declare no conflict of interest.

Keywords: heterogeneous catalysis . nucleophilic substitution - plasmonic nanocavity . self-induced catalysis · spin crossover

When coupling to a nanocavity mode, the molecular dipole moment is the key magnitude dictating how the electronic energy will be affected. In the present case, the dipole moment for the LS state lies along the $\mathrm{Fe}-\mathrm{N}=\mathrm{P}$ axis, while for the HS state, linearity is lost and the dipole moment is practically parallel to the $\mathrm{N}=\mathrm{P}$ direction which is off by approximately $20^{\circ}$ from the $\mathrm{B} \cdots \mathrm{Fe}$ axis. The different occupation of the MOs in both spin states leads to a dipole moment approximately $3 \mathrm{D}$ on average larger in the LS state compared to the HS one, translating into an increase of the electronic energy gap between both states. In Table 1 we show that, when coupling to a EM mode, the $T_{1 / 2}$ values increase around $30 \mathrm{~K}$ on average (with $\lambda=0.02$ ) when we consider the dipole moments of both spin states to be aligned with the polarization of the EM modes. Larger modifications of $T_{1 / 2}$ can be achieved when the dipole moments of both states are perpendicular to each other. Such considerable change proves the success of our proposal as a novel strategy to manipulate molecular properties.

In summary, we have demonstrated how plasmonic nanocavities can modify chemical reaction pathways without the need of external stimuli, neither photonic nor electric. By taking advantage of the strong coupling between the dipole moment of the molecule and the confined EM field associated with plasmonic modes, a self-induced Casimir-Polder force, which critically depends on the reaction coordinate, is able to tailor the ground-state chemical landscape of the molecule. We illustrate this finding by analyzing how the rates of two prototypical $\mathrm{S}_{\mathrm{N}} 1$ and $\mathrm{S}_{\mathrm{N}} 2$ reactions can be altered when the molecules are placed inside a plasmonic nanocavity. In addition, we show how this strategy can also be exploited to manipulate spin states of spin-crossover complexes. This new capability of plasmonic modes to modify molecular properties adds a powerful tool to the long-established and fruitful relation between chemistry and plasmonics.

\section{Acknowledgements}

We are grateful to Dr. Jordi Cirera for advice regarding the modeling of SCO complexes. This work has been funded by the European Research Council (ERC-2016-STG-714870) and the Spanish MINECO under contract MAT2014-53432C5-5-R and the "María de Maeztu" programme for Units of Excellence in R\&D (MDM-2014-0377), as well as through
How to cite: Angew. Chem. Int. Ed. 2019, 58, 8698-8702 Angew. Chem. 2019, 131, 8790-8794

[1] C. R. Hickenboth, J. S. Moore, S. R. White, N. R. Sottos, J. Baudry, S. R. Wilson, Nature 2007, 446, 423-427.

[2] M. T. Ong, J. Leiding, H. Tao, A. M. Virshup, T. J. Martínez, J. Am. Chem. Soc. 2009, 131, 6377-6379.

[3] J. Ribas-Arino, M. Shiga, D. Marx, Angew. Chem. Int. Ed. 2009, 48, 4190-4193; Angew. Chem. 2009, 121, 4254-4257.

[4] W. Quapp, J. M. Bofill, J. Ribas-Ariño, J. Phys. Chem. A 2017, $121,2820-2838$.

[5] S. Shaik, S. P. De Visser, D. Kumar, J. Am. Chem. Soc. 2004, 126, $11746-11749$

[6] R. Meir, H. Chen, W. Lai, S. Shaik, ChemPhysChem 2010, 11, $301-310$.

[7] A. C. Aragonès, N. L. Haworth, N. Darwish, S. Ciampi, N. J. Bloomfield, G. G. Wallace, I. Diez-Perez, M. L. Coote, Nature 2016, 531, 88-91.

[8] S. Shaik, D. Mandal, R. Ramanan, Nat. Chem. 2016, 8, 1091 1098.

[9] S. Ciampi, N. Darwish, H. M. Aitken, I. Díez-Pérez, M. L. Coote, Chem. Soc. Rev. 2018, 47, 5146-5164.

[10] S. D. Fried, S. G. Boxer, Annu. Rev. Biochem. 2017, 86, 387-415.

[11] V. V. Welborn, L. Ruiz Pestana, T. Head-Gordon, Nat. Catal. 2018, 1, 649-655.

[12] P. Christopher, H. Xin, S. Linic, Nat. Chem. 2011, 3, 467.

[13] S. Mukherjee, F. Libisch, N. Large, O. Neumann, L. V. Brown, J. Cheng, J. B. Lassiter, E. A. Carter, P. Nordlander, N. J. Halas, Nano Lett. 2013, 13, 240-247.

[14] Y. Kim, E. Kazuma, Angew. Chem. Int. Ed. 2019, 58, 4800-4808; Angew. Chem. 2019, 131, 4850-4858.

[15] E. Cortés, Science 2018, 362, 28-29.

[16] L. Zhou, D. F. Swearer, C. Zhang, H. Robatjazi, H. Zhao, L. Henderson, L. Dong, P. Christopher, E. A. Carter, P. Nordlander, et al., Science 2018, 362, 69-72.

[17] D. G. Baranov, M. Wersäll, J. Cuadra, T. J. Antosiewicz, T. Shegai, ACS Photonics 2018, 5, 24-42.

[18] R. Ramanan, D. Danovich, D. Mandal, S. Shaik, J. Am. Chem. Soc. 2018, 140, 4354-4362.

[19] S. Shaik, R. Ramanan, D. Danovich, D. Mandal, Chem. Soc. Rev. 2018, 47, 5125-5145.

[20] L.-J. Yu, M. L. Coote, J. Phys. Chem. A 2019, 123, 582-589.

[21] P. Törmä, W. L. Barnes, Rep. Prog. Phys. 2015, 78, 013901.

[22] T. W. Ebbesen, Acc. Chem. Res. 2016, 49, 2403-2412.

[23] J. Feist, J. Galego, F. J. Garcia-Vidal, ACS Photonics 2018, 5, $205-216$.

[24] R. F. Ribeiro, L. A. Martínez-Martínez, M. Du, J. CamposGonzalez-Angulo, J. Yuen-Zhou, Chem. Sci. 2018, 9, 6325-6339.

[25] M. Hertzog, M. Wang, J. Mony, K. Börjesson, Chem. Soc. Rev. 2019, 48, $937-961$. 
[26] A. Thomas, J. George, A. Shalabney, M. Dryzhakov, S. J. Varma, J. Moran, T. Chervy, X. Zhong, E. Devaux, C. Genet, J. A Hutchinson, T. W. Ebbesen, Angew. Chem. Int. Ed. 2016, 55 , 11462-11466; Angew. Chem. 2016, 128, 11634-11638.

[27] A. Thomas, L. Lethuillier-Karl, K. Nagarajan, R. M. A. Vergauwe, J. George, T. Chervy, A. Shalabney, E. Devaux, C. Genet J. Moran, T. W. Ebbesen, Science 2019, 363, 615-619.

[28] J. A. Hutchison, T. Schwartz, C. Genet, E. Devaux, T. W. Ebbesen, Angew. Chem. Int. Ed. 2012, 51, 1592-1596; Angew. Chem. 2012, 124, 1624-1628.

[29] B. Munkhbat, M. Wersäll, D. G. Baranov, T. J. Antosiewicz, T. Shegai, Sci. Adv. 2018, 4, eaas9552.

[30] V. N. Peters, M. O. Faruk, J. Asane, R. Alexander, D. A. Peters, S. Prayakarao, S. Rout, M. A. Noginov, Optica 2019, 6, 318-325.

[31] J. Galego, C. Climent, F. J. Garcia-Vidal, J. Feist, arXiv:1807.10846 2018, $1-22$

[32] J. Flick, H. Appel, M. Ruggenthaler, A. Rubio, J. Chem. Theory Comput. 2017, 13, 1616-1625.

[33] H. B. G. Casimir, D. Polder, Phys. Rev. 1948, 73, 360-372.

[34] P. W. Milonni in Quantum Vacuum (Ed.: P. W. Milonni), Academic Press, San Diego, 1994, pp. 77-114.

[35] C. Genet, F. Intravaia, A. Lambrecht, S. Reynaud, Ann. la Fond. Louis Broglie 2004, 29, $331-348$.
[36] H. B. G. Casimir, J. Chim. Phys. 1949, 46, 407-410.

[37] S. Y. Buhmann, Dispersion Forces I: Macroscopic Quantum Electrodynamics and Ground-State Casimir, Casimir-Polder and van Der Waals Forces, Springer, Berlin, 2012.

[38] J. Clayden, N. Greeves, S. Warren, Organic Chemistry, Oxford University Press, Oxford, 2012.

[39] A. N. Jay, K. A. Daniel, E. V. Patterson, J. Chem. Theory Comput. 2007, 3, 336-343.

[40] P. Gamez, J. S. Costa, M. Quesada, G. Aromí, Dalton Trans. 2009, $7845-7853$.

[41] H. J. Lin, D. Siretanu, D. A. Dickie, D. Subedi, J. J. Scepaniak, D. Mitcov, R. Clérac, J. M. Smith, J. Am. Chem. Soc. 2014, 136, $13326-13332$

[42] J. Cirera, E. Ruiz, Inorg. Chem. 2016, 55, 1657-1663.

[43] G. D. Harzmann, R. Frisenda, H. S. J. van der Zant, M. Mayor, Angew. Chem. Int. Ed. 2015, 54, 13425-13430; Angew. Chem. 2015, 127, 13624-13630

Manuscript received: February 13, 2019

Accepted manuscript online: April 10, 2019

Version of record online: May 21, 2019 